\title{
Revisiting the asymptotic dynamics of General Relativity on $\mathrm{AdS}_{3}$
}

\author{
Hernán A. González, ${ }^{a}$ Javier Matulich, ${ }^{b}$ Miguel Pino ${ }^{c}$ and Ricardo Troncoso ${ }^{d}$ \\ ${ }^{a}$ Institute for Theoretical Physics, TU Wien, \\ Wiedner Hauptstr. 8-10/136, A-1040 Vienna, Austria \\ ${ }^{b}$ Université Libre de Bruxelles and International Solvay Institutes, \\ ULB-Campus Plaine CP231, 1050 Brussels, Belgium \\ ${ }^{c}$ Departamento de Física, Universidad de Santiago de Chile, \\ Avenida Ecuador 3493, Estación Central, 9170124, Santiago, Chile \\ ${ }^{d}$ Centro de Estudios Cientificos (CECs), \\ Av. Arturo Prat 514, Valdivia, Chile \\ E-mail: hgonzale@hep.itp.tuwien.ac.at, jmatulic@ulb.ac.be, \\ miguel.pino.r@usach.cl, troncoso@cecs.cl
}

ABSTRACT: The dual dynamics of Einstein gravity on $\mathrm{AdS}_{3}$ supplemented with boundary conditions of $\mathrm{KdV}$-type is identified. It corresponds to a two-dimensional field theory at the boundary, described by a novel action principle whose field equations are given by two copies of the "potential modified KdV" equation. The asymptotic symmetries then transmute into the global Noether symmetries of the dual action, giving rise to an infinite set of commuting conserved charges, implying the integrability of the system. Noteworthy, the theory at the boundary is non-relativistic and possesses anisotropic scaling of Lifshitz type.

Keywords: Conformal and W Symmetry, Space-Time Symmetries, Gauge-gravity correspondence, Integrable Hierarchies

ARXIV EPRINT: 1809.02749 


\section{Contents}

1 Introduction 1

2 General Relativity on $\mathrm{AdS}_{3}$ and the KdV hierarchy 2

3 Dual theory at the boundary $\quad 6$

3.1 Hamiltonian reduction 6

$3.2 n=0$ : chiral bosons $\quad 9$

$\begin{array}{ll}3.3 n=1: \text { pmKdV movers } & 10\end{array}$

$\begin{array}{ll}3.4 \text { Generic } n \text { : pmKdV hierarchy } & 10\end{array}$

4 Symmetries of the action $\quad 10$

$\begin{array}{lll}4.1 \text { Global symmetries } & 11\end{array}$

4.2 Kinematic symmetries \& Lifshitz scaling 11

5 Concluding remarks $\quad 12$

A Gelfand-Dikii polynomials and Hamiltonians $\quad 14$

$\begin{array}{ll}\text { B pmKdV equations } & 15\end{array}$

\section{Introduction}

The dynamics of Einstein gravity in three spacetime dimensions is described by global degrees of freedom that can be identified only once a precise set of boundary conditions is provided. In the case of asymptotically AdS spacetimes equipped with Brown-Henneaux boundary conditions, the asymptotic symmetry group is generated by two copies of the Virasoro algebra [1]. Demanding that the Lagrange multipliers -given by the lapse and shift functions in an ADM foliation- are held constant at infinity, the reduced phase space of the Einstein field equations is described by Virasoro modes $\mathcal{L}^{ \pm}$that evolve according to

$$
\pm \ell \partial_{t} \mathcal{L}^{ \pm}=\partial_{\phi} \mathcal{L}^{ \pm}
$$

where $\ell$ is the AdS radius and $t, \phi$ are coordinates parametrizing the cylinder at infinity. The symmetry algebra and the form of the latter equation is consistent with the description in terms of the boundary theory; it is well-known that the asymptotic dynamics for these boundary conditions is described by left and right chiral bosons $[2,3] .{ }^{1}$ The components of

\footnotetext{
${ }^{1}$ As shown in [2,3], it is possible to rewrite the action of two chiral bosons as a Liouville theory. This is accomplished by performing a Bäcklund transformation that excludes the zero mode sector of the chiral bosons. Further developments about the dual theory at the boundary have been recently carried out in $[4,5]$.
} 
the stress-energy tensor of the chiral bosons are given by the Virasoro modes $\mathcal{L}^{ \pm}$, so that equation (1.1) corresponds to its conservation law. Note that for the boundary conditions of Brown and Henneaux, the chiral bosons and their corresponding left/right energies fulfill the same equations.

Recently, a new family of boundary conditions connecting Einstein gravity on $\mathrm{AdS}_{3}$ with the Korteweg-de Vries (KdV) hierarchy of integrable systems has been proposed in [6]. The possible choices of boundary conditions are labeled by a nonnegative integer $n$, corresponding to the $n$-th representative of the hierarchy. The Brown-Henneaux boundary conditions are recovered for $n=0$, so that the modes fulfill (1.1); while for $n=1$, the modes are described by noninteracting movers, satisfying the KdV equation

$$
\pm \ell \partial_{t} \mathcal{L}^{ \pm}=3 \mathcal{L}^{ \pm} \partial_{\phi} \mathcal{L}^{ \pm}-\frac{\ell}{16 \pi G} \partial_{\phi}^{3} \mathcal{L}^{ \pm}
$$

where $G$ is the Newton constant. For $n \geq 1$ the asymptotic symmetry algebra turns out to be spanned precisely by the infinite set of commuting charges of KdV.

One of the main purposes of our work, is to unveil the precise form of the action principle that describes the dynamics of the underlying fields of the dual theory at the boundary, from which the field equations of the KdV hierarchy emerge from a conservation law. In order to carry out this task, it is convenient to use the Chern-Simons formulation of three-dimensional gravity $[7,8]$. We then perform a Hamiltonian reduction similar to the one of Coussaert, Henneaux and van Driel [2]. A distinguishing feature of our derivation is that, as the boundary conditions for $n>0$ actually precludes one from passing through the standard Hamiltonian reduction of the Wess-Zumino-Witten (WZW) model [9, 10], one has to circumvent this step through imposing the boundary conditions in the action principle from scratch. In this way, one obtains a novel action principle for the dual theory, whose field equations are described by two copies of the hierarchy of "potential modified KdV" (pmKdV) equations of opposite chirality. ${ }^{2}$

The paper is organized as follows. In the next section, we revisit the boundary conditions of KdV-type in the context of 3D gravity with negative cosmological constant. In section 3, the dual theory at the boundary is obtained from the Hamiltonian reduction of the Chern-Simons action endowed with a suitable boundary term. The field equations are also analyzed. Section 4 is devoted to study the global symmetries of the dual action principle at the boundary. We conclude in with some comments in section 5 .

\section{General Relativity on $\mathrm{AdS}_{3}$ and the KdV hierarchy}

Three-dimensional gravity with negative cosmological constant can be formulated as the difference of two Chern-Simons actions for $\operatorname{sl}(2, \mathbb{R})$-valued gauge fields $A^{ \pm}[7,8]$

$$
I=I_{\mathrm{CS}}\left[A^{+}\right]-I_{\mathrm{CS}}\left[A^{-}\right]
$$

where $I_{\mathrm{CS}}$ reads

$$
I_{\mathrm{CS}}[A]=\frac{k}{4 \pi} \int_{\mathcal{M}}\left\langle A d A+\frac{2}{3} A^{3}\right\rangle
$$

\footnotetext{
${ }^{2} \mathrm{~A}$ list of the first four equations of the pmKdV hierarchy is given in appendix B.
} 
and the Chern-Simons level is given by $k=\frac{\ell}{4 G}$. Here, $\mathcal{M}$ is the three-dimensional manifold with coordinates $t, r, \phi$, where $t$ represents time, $r$ stands for the radial coordinate and $\phi$ is an angle. The generators of the $\operatorname{sl}(2, \mathbb{R})$ algebra, given by $L_{m}^{ \pm}$, with $m=\{-1,0,1\}$, are chosen such that the commutators and the invariant non-degenerate bilinear form read

$$
\left[L_{m}^{ \pm}, L_{n}^{ \pm}\right]=(m-n) L_{m+n}^{ \pm}, \quad\left[L_{m}^{ \pm}, L_{n}^{\mp}\right]=0
$$

and

$$
\left\langle L_{0}^{ \pm 2}\right\rangle=\frac{1}{2}, \quad\left\langle L_{1}^{ \pm} L_{-1}^{ \pm}\right\rangle=-1
$$

respectively.

In order to describe the asymptotic form of the gauge fields, it is useful to make a gauge choice as in [2], so that the connection reads

$$
A^{ \pm}=b_{ \pm}^{-1} a^{ \pm} b_{ \pm}+b_{ \pm}^{-1} d b_{ \pm}
$$

with $b_{ \pm}=e^{ \pm \log (r / l) L_{0}^{ \pm}}$. The components of the auxiliary connection $a^{ \pm}=a_{\phi}^{ \pm} d \phi+a_{t}^{ \pm} d t$, then depend only on time and the angular coordinate, and are generically given by [11, 12]

$$
\begin{aligned}
& a_{\phi}^{ \pm}=L_{ \pm 1}^{ \pm}-\frac{2 \pi}{k} \mathcal{L}^{ \pm} L_{\mp 1}^{ \pm}, \\
& a_{t}^{ \pm}= \pm \frac{1}{\ell} \mu^{ \pm} L_{ \pm 1}^{ \pm}-\frac{1}{\ell} \partial_{\phi} \mu^{ \pm} L_{0}^{ \pm} \pm\left(\frac{1}{2 \ell} \partial_{\phi}^{2} \mu^{ \pm}-\frac{2 \pi}{k \ell} \mathcal{L}^{ \pm} \mu^{ \pm}\right) L_{\mp 1}^{ \pm},
\end{aligned}
$$

where $\mathcal{L}^{ \pm}(t, \phi)$ stand for the dynamical fields, and $\mu^{ \pm}(t, \phi)$ correspond to the Lagrange multipliers. In the asymptotic region, the field equations, $F^{ \pm}=d A^{ \pm}+A^{ \pm} \wedge A^{ \pm}=0$, reduce to

$$
\pm \ell \partial_{t} \mathcal{L}^{ \pm}=\mathcal{D}^{ \pm} \mu^{ \pm}
$$

where the operators $\mathcal{D}^{ \pm}$are defined by

$$
\mathcal{D}^{ \pm} \equiv\left(\partial_{\phi} \mathcal{L}^{ \pm}\right)+2 \mathcal{L}^{ \pm} \partial_{\phi}-\frac{k}{4 \pi} \partial_{\phi}^{3} .
$$

The asymptotic symmetries can then be explicitly found by demanding the preservation of the auxiliary connection $a^{ \pm}$under gauge transformations, $\delta a^{ \pm}=d \eta^{ \pm}+\left[a^{ \pm}, \eta^{ \pm}\right]$, where $\eta^{ \pm}$is a Lie-algebra-valued parameter. Thus, the asymptotic form of $a_{\phi}$ is maintained for gauge transformations spanned by parameters of the form

$$
\eta^{ \pm}=\varepsilon^{ \pm} L_{ \pm 1}^{ \pm} \mp \partial_{\phi} \varepsilon^{ \pm} L_{0}^{ \pm}+\left(\frac{1}{2} \partial_{\phi}^{2} \varepsilon^{ \pm}-\frac{2 \pi}{k} \mathcal{L}^{ \pm} \varepsilon^{ \pm}\right) L_{\mp 1}^{ \pm},
$$

where $\varepsilon^{ \pm}$are arbitrary functions of $t$ and $\phi$, provided that the dynamical fields $\mathcal{L}^{ \pm}$transform as

$$
\delta \mathcal{L}^{ \pm}=\mathcal{D}^{ \pm} \varepsilon^{ \pm} .
$$

Preserving the temporal component of the gauge field $a_{t}^{ \pm}$then implies the following condition for the variation of the Lagrange multipliers

$$
\delta \mu^{ \pm}= \pm \ell \partial_{t} \varepsilon^{ \pm}+\partial_{\phi} \mu^{ \pm} \varepsilon^{ \pm}-\mu^{ \pm} \partial_{\phi} \varepsilon^{ \pm}
$$


It is worth stressing that the boundary conditions turn out to be fully determined only once the precise form of the Lagrange multipliers at the boundary is specified. The results of Brown and Henneaux [1] are then recovered when the Lagrange multipliers are held constants at infinity $\mu^{ \pm}=1$. A simple generalization is obtained by choosing arbitrary functions of the coordinates, so that $\mu^{ \pm}=\mu^{ \pm}(t, \phi)$ are kept fixed at the boundary $\left(\delta \mu^{ \pm}=0\right)[11,12]$. Different choices of boundary conditions, in which the Lagrange multipliers are allowed to depend on the dynamical fields and their spatial derivatives, were proposed in [6]. Hereafter, we focus in a special family of boundary conditions of KdVtype, being labeled by a non negative integer $n$. In this scenario, the Lagrange multipliers are chosen to be given by the $n$-th Gelfand-Dikii polynomial [13] evaluated on $\mathcal{L}^{ \pm}$, i.e.,

$$
\mu^{ \pm} \equiv \mu_{n}^{ \pm}=R_{n}^{ \pm}\left[\mathcal{L}^{ \pm}\right]
$$

The polynomials can be constructed by means of the following recursion relation ${ }^{3}$

$$
\mathcal{D}^{ \pm} R_{n}^{ \pm}=\partial_{\phi} R_{n+1}^{ \pm}
$$

Thus, in the case of $n=0$ one obtains that $\mu_{0}^{ \pm}=R_{0}^{ \pm}=1$, which reduces to the boundary conditions of Brown and Henneaux [1]. In this case, equation (2.11) implies that the parameters $\varepsilon^{ \pm}$are chiral, while the dynamical fields also do, since the field equations $(2.7)$ reduce to (1.1). The next case corresponds to $n=1$, so that the choice of Lagrange multipliers is given by $\mu_{1}^{ \pm}=R_{1}^{ \pm}=\mathcal{L}^{ \pm}$, and hence, the field equations in (2.7) reduce to $\mathrm{KdV}$

$$
\pm \ell \partial_{t} \mathcal{L}^{ \pm}=3 \mathcal{L}^{ \pm} \partial_{\phi} \mathcal{L}^{ \pm}-\frac{k}{4 \pi} \partial_{\phi}^{3} \mathcal{L}^{ \pm}
$$

In the remaining cases, $n>1$, the field equations are then given by the ones of the $n$-th representative of the $\mathrm{KdV}$ hierarchy.

Note that for $n>0$, the Lagrange multipliers acquire a non-trivial variation at infinity. Nonetheless, as shown in [6] and further explained in the next section, the action principle can be well defined because each of the Gelfand-Dikii polynomials $R_{n}^{ \pm}$can be expressed in terms of the variation of a functional, i.e.,

$$
R_{n}^{ \pm}=\frac{\delta H_{n}^{ \pm}}{\delta \mathcal{L}^{ \pm}}, \quad H_{n}^{ \pm}=\int d \phi \mathcal{H}_{n}^{ \pm}
$$

where $H_{n}^{ \pm}$stand for the conserved quantities of $\mathrm{KdV}$, and $\mathcal{H}_{n}^{ \pm}$are the corresponding densities. ${ }^{4}$ Furthermore, equation (2.11) becomes a consistency relation for the time derivative of the asymptotic symmetry parameters $\varepsilon^{ \pm}$. Thus, for $n \geq 1$, assuming that the parameters depend exclusively on the dynamical fields and their spatial derivatives, but not explicitly on the coordinates, the general solution of the consistency relation is given by a linear combination of the form

$$
\varepsilon^{ \pm}=\sum_{j=0}^{\infty} \epsilon_{j}^{ \pm} R_{j}^{ \pm},
$$

\footnotetext{
${ }^{3}$ Note that the normalization of the Gelfand-Dikii polynomials used here differs from the one in [6].

${ }^{4} \mathrm{~A}$ list with the first Gelfand-Dikii polynomials, conserved quantities of $\mathrm{KdV}$ and the corresponding field equations of the KdV hierarchy is given in appendix A.
} 
with $\epsilon_{j}^{ \pm}$constants. This infinite set of symmetries then gives rise to conserved charges, which can be written as surface integrals by means of the Regge-Teitelboim approach [14]. The variation of the conserved charges associated to the gauge transformation generated by a parameter of the form (2.9) that spans the asymptotic symmetries, is given by

$$
\delta Q\left[\varepsilon^{ \pm}\right]=\mp \int d \phi \varepsilon^{ \pm} \delta \mathcal{L}^{ \pm}
$$

which can be integrated due to (2.16) and (2.15), yielding

$$
Q\left[\varepsilon^{ \pm}\right]=\mp \sum_{j=0}^{\infty} \epsilon_{j}^{ \pm} H_{j}^{ \pm} .
$$

The asymptotic symmetries are then canonically realized. A straightforward way to obtain the asymptotic symmetry algebra in terms of Poisson brackets is given by the relation

$$
\left\{Q\left[\varepsilon_{1}\right], Q\left[\varepsilon_{2}\right]\right\}=\delta_{\varepsilon_{2}} Q\left[\varepsilon_{1}\right] .
$$

The cases $n=0$ and $n>0$ are then very different in this context. Indeed, for $n>0$ the algebra turns out to be abelian

$$
\left\{H_{i}^{ \pm}, H_{j}^{ \pm}\right\}=0,
$$

while for $n=0$, which corresponds to Brown-Henneaux, the algebra of the conserved charges is given by two copies of the Virasoro algebra with a non-vanishing central extension.

Some interesting remarks about the metric formulation are in order. It is worth highlighting that the reduced phase space for the boundary conditions of KdV-type, for an arbitrary non negative integer $n$, always contain the BTZ black hole $[15,16]$, which corresponds to the configuration with $\mathcal{L}^{ \pm}$constants [6]. Indeed, the field equations of the $\mathrm{KdV}$ hierarchy are trivially solved in this case, and the spacetime metric in the ADM decomposition is such that the lapse and the shift correspond to a non-standard foliation, determined by $\mu_{n}^{ \pm}=\frac{(2 n) !}{2^{n}(n !)^{2}}\left(\mathcal{L}^{ \pm}\right)^{n}$. Specifically

$$
d s^{2}=\ell^{2}\left[\frac{d r^{2}}{r^{2}}+\frac{2 \pi}{k} \mathcal{L}^{+}\left(d \widetilde{x}^{+}\right)^{2}+\frac{2 \pi}{k} \mathcal{L}^{-}\left(d \widetilde{x}^{-}\right)^{2}-\left(\frac{r^{2}}{\ell^{2}}+\left(\frac{2 \pi \ell}{k}\right)^{2} \frac{\mathcal{L}^{+} \mathcal{L}^{-}}{r^{2}}\right) d \widetilde{x}^{+} d \widetilde{x}^{-}\right],
$$

with

$$
d \widetilde{x}^{ \pm}=\frac{1}{\ell} \mu_{n}^{ \pm} d t \pm d \varphi
$$

Furthermore, the boundary conditions described by (2.5) and (2.6), with $\mu_{n}^{ \pm}$given by (2.12) are such that the fall-off of the metric somewhat resembles the one of Brown-Henneaux. Indeed, in a Fefferman-Graham-like gauge, the spatial components of the metric and its conjugate momenta behave as

$$
\begin{array}{lll}
g_{r r}=\frac{\ell^{2}}{r^{2}}, & g_{r \phi}=0, & g_{\phi \phi}=r^{2}+O(1), \\
\pi^{r r}=O\left(r^{-1}\right), & \pi^{r \phi}=O\left(r^{-2}\right), & \pi^{\phi \phi}=0 .
\end{array}
$$


However, the key difference arises in the asymptotic behavior of the lapse and shift functions, which read

$$
\begin{aligned}
N^{\perp} & =\frac{1}{2}\left(\mu_{n}^{+}+\mu_{n}^{-}\right) \frac{r}{\ell}+O\left(r^{-1}\right), \\
N^{r} & =-\left(\partial_{\phi} \mu_{n}^{+}-\partial_{\phi} \mu_{n}^{-}\right) \frac{r}{2 \ell}+O\left(r^{-2}\right), \\
N^{\phi} & =\frac{1}{2 \ell}\left(\mu_{n}^{+}-\mu_{n}^{-}\right)+O\left(r^{-2}\right) .
\end{aligned}
$$

Hence, for $n>0$ they are allowed to fluctuate at leading order, in sharp contrast with the fall-off for $n=0$ that corresponds to the Brown-Henneaux boundary conditions for which $\mu_{0}^{+}=\mu_{0}^{-}=1$.

\section{Dual theory at the boundary}

In this section, we perform a Hamiltonian reduction of the action (2.1) by explicitly solving the constraints of the theory. The boundary conditions for the gauge field $A^{ \pm}$correspond to (2.5) and (2.6), where the "chemical potentials" $\mu^{ \pm}$in (2.12) are given by the $n$-th Gelfand-Dikii polynomial $R_{n}^{ \pm}\left[\mathcal{L}^{ \pm}\right]$. The reduction is carried out for a generic value of $n$.

\subsection{Hamiltonian reduction}

The Hamiltonian reduction of Chern-Simons theory in the context of three-dimensional gravity has been discussed extensively in the literature, see e.g., [2, 3, 17, 18]. For the standard choices of boundary conditions $[1,19]$, the classical dynamics can be obtained from the Hamiltonian reduction of the WZW theory at the boundary [9, 10, 20, 21]. Nonetheless, for the boundary conditions of KdV-type, the reduction does not lead to the usual WZW theory at the boundary, since for a generic value of $n$ the components of the gauge field at the boundary are no longer proportional, and hence, the Kac-Moody symmetry appears to be manifestly broken (except when $n=0$ which corresponds to Brown-Henneaux). Nevertheless, as explained below, the reduction can still be successfully performed because the boundary conditions can be appropriately implemented in the action principle.

The resulting reduced action at the boundary gives rise to a different hierarchy of integrable equations, labeled by the integer $n$. The simplest case $(n=0)$ corresponds to two chiral bosons of opposite chirality $[2,3]$. For $n=1$ we obtain a novel action principle, whose field equations are given by two copies of the pmKdV equation (see e.g. [22, 23]). In the remaining cases $(n>1)$ the action of the dual theory describes the other members of the pmKdV hierarchy. The integrability of this hierarchy is explicitly checked in the next section.

We start with the action (2.1) written in explicit Hamiltonian form

$$
I=I_{H}\left[A^{+}\right]-I_{H}\left[A^{-}\right],
$$

with

$$
I_{H}\left[A^{ \pm}\right]=\frac{k}{4 \pi} \int d t d^{2} x \epsilon^{i j}\left\langle\dot{A}_{i}^{ \pm} A_{j}^{ \pm}+A_{t}^{ \pm} F_{i j}^{ \pm}\right\rangle+B^{ \pm},
$$


where $B^{ \pm}$stand for appropriate boundary terms generically needed in order to have an action principle that is well defined. It is worth pointing out that the boundary can be located at an arbitrary fixed value of the radial coordinate. Here $\epsilon^{i j}$ is the spatial part of the Levi-Civita symbol, while $F_{i j}^{ \pm}$is the curvature $F_{i j}^{ \pm}=\partial_{i} A_{j}^{ \pm}-\partial_{j} A_{i}^{ \pm}+\left[A_{i}^{ \pm}, A_{j}^{ \pm}\right]$. We choose $\epsilon^{r \phi}=1$, and dot stands for derivative with respect to $t$. The action (3.2) attains an extremum when the field equations hold, provided that

$$
\delta B^{ \pm}=-\frac{k}{2 \pi} \int d \phi d t\left\langle A_{t}^{ \pm} \delta A_{\phi}^{ \pm}\right\rangle .
$$

Note that for the Brown-Henneaux boundary conditions $(n=0)$, the components of the gauge field satisfy $\ell A_{t}^{ \pm}=A_{\phi}^{ \pm}$at the boundary, and hence, $\delta B^{ \pm}$can be readily integrated. However, for the boundary conditions of KdV-type, with $n \geq 1$, the temporal and angular components of the gauge field at the boundary are not proportional (see (2.6)), and so one might worry about the integrability of the boundary terms $B^{ \pm}$. However, as explained in [6], since the Lagrange multipliers $\mu^{ \pm}$in (2.12) are given by the variation of a functional (see $(2.15)$ ) the boundary terms can be explicitly integrated as

$$
B^{ \pm}=\mp \frac{1}{\ell} \int d \phi d t \mathcal{H}_{n}^{ \pm}
$$

Therefore, the suitable action principle for the boundary conditions of KdV-type is precisely identified, and so we are able to proceed with its Hamiltonian reduction.

The constraint $\epsilon^{i j} F_{i j}^{ \pm}=0$ is locally solved by $A_{i}^{ \pm}=G_{ \pm}^{-1} \partial_{i} G_{ \pm}$. For the sake of simplicity, we disregard non-trivial holonomies, so that $G_{ \pm}(t, r, \phi) \in \mathrm{SL}(2, \mathbb{R})$ can be assumed to be periodic in $\phi$. Thus, replacing back in the action (3.2), a straightforward calculation yields

$$
I_{H}\left[A^{ \pm}\right]=I_{1}^{ \pm}+I_{2}^{ \pm}+B^{ \pm}
$$

where

$$
\begin{aligned}
& I_{1}^{ \pm}=\frac{k}{4 \pi} \int d t d r d \phi \epsilon^{i j}\left\langle\partial_{t}\left(G_{ \pm}^{-1}\right) \partial_{i} G_{ \pm} G_{ \pm}^{-1} \partial_{j} G_{ \pm}\right\rangle, \\
& I_{2}^{ \pm}=-\frac{k}{4 \pi} \int d \phi d t\left\langle\partial_{t} G_{ \pm} \partial_{\phi}\left(G_{ \pm}^{-1}\right)\right\rangle .
\end{aligned}
$$

The first two terms $I_{1}^{ \pm}+I_{2}^{ \pm}$naturally appear in the standard chiral WZW action [21], but here we have an explicit modification due to the presence of $B^{ \pm}$. As shown below, the form of $B^{ \pm}$makes possible to recover the infinite-dimensional Abelian algebra in (2.20) from a Noether symmetry of the full action. Furthermore, note that $B^{ \pm}$do not appear to be expressible locally in terms of the group elements $G_{ \pm}$.

In order to reduce $I_{1}^{ \pm}$to a boundary integral, we use the Gauss decomposition for $G_{ \pm}$

$$
G_{ \pm}=\exp \left[X_{ \pm} L_{ \pm 1}^{ \pm}\right] \exp \left[ \pm \Phi_{ \pm} L_{0}^{ \pm}\right] \exp \left[Y_{ \pm} L_{\mp 1}^{ \pm}\right] .
$$

Here $X_{ \pm}, Y_{ \pm}$and $\Phi_{ \pm}$are functions of $t, r, \phi$. Thus, $I_{1}^{ \pm}$can be expressed as

$$
I_{1}^{ \pm}=\frac{k}{4 \pi} \int d \phi d t e^{\Phi_{ \pm}}\left(Y_{ \pm}^{\prime} \dot{X}_{ \pm}-\dot{Y}_{ \pm} X_{ \pm}^{\prime}\right)
$$


where prime denotes derivatives with respect to $\phi$. Thus, the action $I_{H}\left[A^{ \pm}\right]$has now been reduced to an integral at the boundary.

The fall-off in (2.5), (2.6) allows us to decompose the group element $G_{ \pm}$in the asymptotic region as a product of two group elements, according to $G_{ \pm}(t, r, \phi)=g_{ \pm}(t, \phi) b_{ \pm}(r)$, with $b_{ \pm}=e^{ \pm \log (r / \ell) L_{0}^{ \pm}}$. Therefore, $I_{2}^{ \pm}$reduces to

$$
I_{2}^{ \pm}=-\frac{k}{4 \pi} \int d \phi d t\left\langle\dot{g}_{ \pm} \partial_{\phi} g_{ \pm}^{-1}\right\rangle
$$

which can be further simplified by performing the Gauss decomposition for the group element $g_{ \pm}$

$$
g_{ \pm}=e^{x_{ \pm} L_{ \pm 1}^{ \pm}} e^{ \pm \varphi_{ \pm} L_{0}^{ \pm}} e^{y_{ \pm} L_{\text {干 }}^{ \pm}},
$$

where the fields $x_{ \pm}, y_{ \pm}$and $\varphi_{ \pm}$depend only on $t$ and $\phi$. Thus, we obtain

$$
I_{2}^{ \pm}=\frac{k}{4 \pi} \int d \phi d t\left(\frac{1}{2} \dot{\varphi}_{ \pm} \varphi_{ \pm}^{\prime}-e^{\varphi_{ \pm}}\left(y_{ \pm}^{\prime} \dot{x}_{ \pm}+\dot{y}_{ \pm} x_{ \pm}^{\prime}\right)\right)
$$

Furthermore, consistency of (3.8) and (3.11) yields

$$
Y_{ \pm}=\frac{\ell}{r} y_{ \pm}, \quad X_{ \pm}=x_{ \pm}, \quad e^{\Phi_{ \pm}}=\frac{r}{\ell} e^{\varphi_{ \pm}}
$$

and hence, the action (3.5) reduces to

$$
I_{H}\left[A^{ \pm}\right]=\frac{k}{4 \pi} \int d \phi d t\left(\frac{1}{2} \dot{\varphi}_{ \pm} \varphi_{ \pm}^{\prime}-2 e^{\varphi_{ \pm}} x_{ \pm}^{\prime} \dot{y}_{ \pm}\right) \mp \frac{1}{\ell} \int d \phi d t \mathcal{H}_{n}^{ \pm}
$$

Besides, the asymptotic form of $a_{\phi}^{ \pm}=g_{ \pm}^{-1} \partial_{\phi} g_{ \pm}$is determined by eq. (2.6), so that

$$
g_{ \pm}^{-1} \partial_{\phi} g_{ \pm}=L_{ \pm 1}^{ \pm}-\frac{2 \pi}{k} \mathcal{L}^{ \pm} L_{\mp 1}^{ \pm}
$$

which by virtue of the Gauss decomposition (3.11), implies the following relations

$$
e^{\varphi_{ \pm}} x_{ \pm}^{\prime}=1, \quad 2 e^{\varphi_{ \pm}} y_{ \pm} x_{ \pm}^{\prime}+\varphi_{ \pm}^{\prime}=0, \quad y_{ \pm}^{\prime}+e^{\varphi_{ \pm}} y_{ \pm}^{2} x_{ \pm}^{\prime}+y_{ \pm} \varphi_{ \pm}^{\prime}=-\frac{2 \pi}{k} \mathcal{L}^{ \pm} .
$$

Making use of the first equation in (3.16), it is straightforward to see that the second term in (3.14) becomes a total time derivative that can be discarded. The remaining equations in (3.16) then allow to obtain a crucial relationship, given by

$$
\mathcal{L}^{ \pm}=\frac{k}{4 \pi}\left(\frac{1}{2} \varphi_{ \pm}^{\prime 2}+\varphi_{ \pm}^{\prime \prime}\right)
$$

from which the reduced action (3.14) can be expressed exclusively in term of two fundamental fields $\varphi_{ \pm}$.

In sum, the action of the dual theory at the boundary explicitly reads

$$
I_{n}\left[\varphi_{+}, \varphi_{-}\right]=I_{n}\left[\varphi_{+}\right]-I_{n}\left[\varphi_{-}\right],
$$


with

$$
I_{n}\left[\varphi_{ \pm}\right]=\frac{k}{8 \pi} \int d \phi d t\left(\dot{\varphi}_{ \pm} \varphi_{ \pm}^{\prime} \mp \frac{8 \pi}{\ell k} \mathcal{H}_{n}^{ \pm}\left[\mathcal{L}^{ \pm}\right]\right)
$$

where $\mathcal{L}^{ \pm}$is given by (3.17). A complete analysis of the global symmetries of the dual action (3.19) is performed in section 4 .

For the remaining steps, it is worth highlighting that the action (3.19) possesses the following gauge symmetry

$$
\varphi_{ \pm} \rightarrow \varphi_{ \pm}+f_{ \pm}(t)
$$

where $f_{ \pm}$stand for arbitrary functions. Indeed, under (3.20), the kinetic term in (3.19) just changes by a time derivative, while the Hamiltonian does not give additional contributions since $\mathcal{H}_{n}^{ \pm}$only involves angular derivatives of $\varphi_{ \pm}$.

In order to familiarize with the dynamics of the theory at the boundary, it is certainly useful to analyze its field equations. As it is shown below, the gauge freedom in (3.20) allows to write down the equations of motion of (3.19) precisely as those of the pmKdV hierarchy.

\section{$3.2 n=0$ : chiral bosons}

The simplest case is given by $\mu_{0}^{ \pm}=1$, which corresponds to the Brown-Henneaux boundary conditions. The dual theory is obtained from (3.18) and (3.19) with $n=0$, so that $\mathcal{H}_{0}^{ \pm}=\mathcal{L}^{ \pm}$, and hence each copy of the action reduces to the one of Floreanini and Jackiw [24]

$$
I_{0}\left[\varphi_{ \pm}\right]=\frac{k}{8 \pi} \int d \phi d t\left(\dot{\varphi}_{ \pm} \varphi_{ \pm}^{\prime} \mp \frac{1}{\ell} \varphi_{ \pm}^{2}\right)
$$

in agreement with the standard result obtained in $[2,3]$. The theory describes the dynamics of two chiral bosons of opposite chirality. The field equations in this case then read

$$
\dot{\varphi}_{ \pm}^{\prime}= \pm \frac{1}{\ell} \varphi_{ \pm}^{\prime \prime},
$$

which can be readily integrated once, yielding

$$
\dot{\varphi}_{ \pm}= \pm \frac{1}{\ell} \varphi_{ \pm}^{\prime}+h_{ \pm}(t),
$$

where $h_{ \pm}$are arbitrary functions of time. Therefore, these arbitrary functions can be set to zero by virtue of the gauge symmetry in $(3.20)$, with $h_{ \pm}(t)=\dot{f}_{ \pm}(t)$, and hence

$$
\dot{\varphi}_{ \pm}= \pm \frac{1}{\ell} \varphi_{ \pm}^{\prime}
$$

Note that, as mentioned in the introduction, the field equations for $n=0$ in (3.24) coincide with the ones of the Virasoro modes in (1.1). As it is shown below, in our context, the fact that the field equation is equivalent to the conservation law it is actually an accident of the particular case $n=0$. 


\section{$3.3 n=1:$ pmKdV movers}

The next case corresponds to the choice $\mu_{1}^{ \pm}=\mathcal{L}^{ \pm}$so that $\mathcal{H}_{1}^{ \pm}=\frac{1}{2} \mathcal{L}^{ \pm 2}$. The chiral copies of the action then read

$$
I_{1}\left[\varphi_{ \pm}\right]=\frac{k}{8 \pi} \int d \phi d t\left(\dot{\varphi}_{ \pm} \varphi_{ \pm}^{\prime} \mp \frac{k}{16 \pi \ell} \varphi_{ \pm}^{\prime 4} \mp \frac{k}{4 \pi \ell} \varphi_{ \pm}^{\prime \prime 2}\right)
$$

and the field equations are given by

$$
\dot{\varphi}_{ \pm}^{\prime}= \pm \frac{k}{4 \pi \ell}\left(\frac{3}{2} \varphi_{ \pm}^{\prime}{ }^{2} \varphi_{ \pm}^{\prime \prime}-\varphi_{ \pm}^{\prime \prime \prime \prime}\right)
$$

As in the previous case, the equation can be integrated once, giving

$$
\dot{\varphi}_{ \pm}= \pm \frac{k}{4 \pi \ell}\left(\frac{1}{2} \varphi_{ \pm}^{\prime 3}-\varphi_{ \pm}^{\prime \prime \prime}\right)
$$

where the arbitrary integration function has been set to zero by virtue of an appropriate gauge choice. This equation corresponds to two copies of the pmKdV equation. ${ }^{5}$

It is worth highlighting that in this case the dynamics of the theory at the boundary, described by (3.27), differs from the conservation law that fulfills the KdV equation (1.2).

\subsection{Generic $n$ : pmKdV hierarchy}

The generic choice of boundary conditions corresponds to $\mu_{n}^{ \pm}=R_{n}^{ \pm}=\frac{\delta H_{n}^{ \pm}}{\delta \mathcal{L}^{ \pm}}$, where $R_{n}^{ \pm}$ stand for the $n$-th Gelfand-Dikii polynomials. The action is then given by (3.18) and (3.19) with (3.17).

The field equations can be readily obtained in a closed form for a generic value of $n$, yielding

$$
\dot{\varphi}_{ \pm}^{\prime}= \pm \frac{1}{\ell} \partial_{\phi}\left(R_{n}^{ \pm} \varphi_{ \pm}^{\prime}-\partial_{\phi} R_{n}^{ \pm}\right) .
$$

As in the previous cases, these equations can be integrated once, and by means of the gauge symmetry of the action (3.20), they reduce to

$$
\dot{\varphi}_{ \pm}= \pm \frac{1}{\ell}\left(R_{n}^{ \pm} \varphi_{ \pm}^{\prime}-\partial_{\phi} R_{n}^{ \pm}\right)
$$

in agreement with $n$-th representative of the potential form of the mKdV hierarchy.

In the next section it is shown that these equations can be manifestly seen to be integrable, since they admit an infinite number of commuting conserved charges.

\section{Symmetries of the action}

This section is devoted to study the symmetries and conserved quantities of the action (3.19). Apart from the gauge symmetry (3.20), the action (3.18) also possesses global and kinematic symmetries, which are described in what follows.

\footnotetext{
${ }^{5}$ The name stems from the fact that under the identification $u=\varphi^{\prime}$, equation (3.26) reduces to modified $\mathrm{KdV}(\mathrm{mKdV})$ for $u$.
} 


\subsection{Global symmetries}

Here we show that the action (3.18) is invariant under the following Noether symmetries

$$
\delta \varphi_{ \pm}=\varepsilon^{ \pm} \varphi_{ \pm}^{\prime}-\varepsilon^{\prime \pm},
$$

with $\varepsilon^{ \pm}$given by (2.16). It is worth stressing that these global symmetries are in one to one correspondence with the asymptotic symmetries in the bulk. Indeed, by means of the map in (3.17), the transformation law of $\mathcal{L}^{ \pm}$that is given by (2.10) is precisely recovered from (4.1). Therefore, the corresponding infinite number of commuting Noether charges $H_{n}^{ \pm}$ can be seen to coincide with the surface integrals that come from the analysis in the bulk.

This can be explicitly shown as follows. For each copy of the action (3.18), the Hamiltonian is invariant under the transformation (4.1), while the kinetic term changes by a total derivative in time. Indeed, equations (2.10) and (2.16), imply that the variation of the Hamiltonian term can be expressed as

$$
\delta \int d \phi d t \mathcal{H}_{n}^{ \pm}=\sum_{k=0}^{\infty} \epsilon_{k}^{ \pm} \int d \phi d t \frac{\delta H_{n}^{ \pm}}{\delta \mathcal{L}^{ \pm}} \mathcal{D}^{ \pm} \frac{\delta H_{k}^{ \pm}}{\delta \mathcal{L}^{ \pm}} .
$$

Thus, the r.h.s. corresponds to the Poisson bracket $\left\{H_{n}^{ \pm}, H_{k}^{ \pm}\right\}$, which vanishes due to (2.13). The variation of the kinetic term in (3.18) reduces to

$$
\delta \frac{k}{8 \pi} \int d \phi d t \dot{\varphi}_{ \pm} \varphi_{ \pm}^{\prime}=\int d \phi d t\left(-\sum_{k=0}^{\infty} \epsilon_{k}^{ \pm} \dot{\mathcal{H}}_{k}^{ \pm}+\frac{k}{8 \pi} \partial_{t}\left(\delta \varphi_{ \pm} \varphi_{ \pm}^{\prime}\right)\right),
$$

and hence the transformation (4.1) is a symmetry of the action. Therefore, the straightforward application of Noether's theorem yields an infinite number of commuting conserved charges given by

$$
Q\left(\varepsilon^{ \pm}\right)=\mp \sum_{k=0}^{\infty} \epsilon_{k}^{ \pm} H_{k}^{ \pm},
$$

which implies that the field equations in (3.29) correspond to an integrable system. Besides, and noteworthy, the Noether charges associated with the global symmetries of the dual theory in (4.4) precisely agree with the surface integrals found from the asymptotic symmetries in the bulk (2.18).

\subsection{Kinematic symmetries \& Lifshitz scaling}

The kinematic symmetries of the dual action (3.18) correspond to rigid displacements in space and time, as well as global anisotropic scaling. These symmetries are spanned by a two-dimensional vector field

$$
X=\left(\alpha^{t}+\gamma z t\right) \partial_{t}+\left(\alpha^{\phi}+\gamma \phi\right) \partial_{\phi},
$$

with $\alpha^{t}, \alpha^{\phi}$ and $\gamma$ constants, and $z$ is related to the integer $n$ through $z=2 n+1$. Under an infinitesimal diffeomorphism spanned by $X$ the scalar fields transform as $\delta_{X} \varphi_{ \pm}=X^{\mu} \partial_{\mu} \varphi_{ \pm}$, which implies that left and right Hamiltonian densities change according to

$$
\delta_{X} \mathcal{H}_{n}^{ \pm}\left[\mathcal{L}^{ \pm}\right]=X^{\mu} \partial_{\mu} \mathcal{H}_{n}^{ \pm}+\gamma(z+1) \mathcal{H}_{n}^{ \pm},
$$

so that under anisotropic scaling they have weight given by $2 n+2$. 
It is simple to prove that the dual action (3.19) is invariant under the symmetries spanned by $X$, and hence, the corresponding Noether charges for the chiral copies are given by

$$
Q^{ \pm}\left[\alpha^{t}, \alpha^{\phi}, \gamma\right]=\int d \phi\left[\left(\alpha^{\phi}+\gamma \phi\right) \mathcal{L}^{ \pm} \pm \frac{1}{\ell}\left(\alpha^{t}+\gamma z t\right) \mathcal{H}_{n}^{ \pm}\right]
$$

Thus, for each copy, the energy, the momentum, and the conserved charge associated to anisotropic scaling are given by

$$
H^{ \pm}=Q^{ \pm}[1,0,0]= \pm \frac{1}{\ell} H_{n}^{ \pm}, \quad P^{ \pm}=Q^{ \pm}[0,1,0]=H_{0}^{ \pm}, \quad D^{ \pm}=Q^{ \pm}[0,0,1],
$$

respectively. Note that $D^{ \pm}$correspond to left and right copies of the generator of anisotropic Lifshitz scaling of the form,

$$
t \mapsto \lambda^{z} t, \quad \phi \mapsto \lambda \phi,
$$

so that $z=2 n+1$ stands for the dynamical exponent. For each copy, the generators of the kinematic symmetry then fulfill the Lifshitz algebra in two dimensions (see e.g. [25-27]). In fact, as it can be readily obtained from relation (2.19), the algebra of the kinematic symmetries reads ${ }^{6}$

$$
\left\{P^{ \pm}, H^{ \pm}\right\}=0, \quad\left\{D^{ \pm}, P^{ \pm}\right\}=P^{ \pm}, \quad\left\{D^{ \pm}, H^{ \pm}\right\}=z H^{ \pm} .
$$

In summary, the only non-vanishing commutators of the infinite set of global symmetries are given by

$$
\left\{D^{ \pm}, H_{j}^{ \pm}\right\}=(2 j+1) H_{j}^{ \pm},
$$

which means that the conserved charges $H_{j}^{ \pm}$transform with weight $2 j+1$ under anisotropic scaling. Thus, the dual action (3.18) is manifestly invariant under (4.9), since the densities transform as $\mathcal{H}_{j}^{ \pm} \mapsto \lambda^{-(2 j+2)} \mathcal{H}_{j}^{ \pm}$.

\section{Concluding remarks}

We have performed a Hamiltonian reduction of General Relativity in 3D with negative cosmological constant in the case of a new family of boundary conditions, labeled by a non negative integer $n$, which is related to the $\mathrm{KdV}$ hierarchy of integrable systems. We then obtained the action of the corresponding dual theory at the boundary, being such that the chiral copies of the reduced system evolve according to the potential form of the modified Korteweg-de Vries equation (3.29).

The asymptotic symmetries in the bulk are then translated into Noether symmetries of the dual theory, giving rise to an infinite set of commuting conserved charges, that imply

\footnotetext{
${ }^{6}$ In order to recover the Lifshitz algebra, it is useful to make use of the following identity [13, 28, 29]:$$
(2 n+1) H_{n}^{ \pm}=\int d \phi R_{n+1}^{ \pm} .
$$ 
integrability of the system. Remarkably, the dual action is also invariant under anisotropic Lifshitz scaling with dynamical exponent $z=2 n+1$.

It is worth pointing out that, if left and right copies were chosen according to different members of the hierarchy, the dual action turns out to be given by

$$
I\left[\varphi_{+}, \varphi_{-}\right]=\int d \phi d t\left(\frac{\ell}{32 \pi G}\left(\dot{\varphi}_{+} \varphi_{+}^{\prime}-\dot{\varphi}_{-} \varphi_{-}^{\prime}\right)-\mathcal{H}_{n}^{+}\left[\mathcal{L}^{+}\right]-\mathcal{H}_{m}^{+}\left[\mathcal{L}^{-}\right]\right)
$$

where $\mathcal{L}^{ \pm}$is defined in (3.17). The anisotropic scaling symmetry would then be generically broken unless $n=m$.

It is interesting to make an interpretation of our work in the context of the fluid/gravity correspondence [30-32]. In that setup, the asymptotic behavior of the Einstein equations, in a derivative expansion at the boundary, implies that the fluid equations are recovered from the conservation of the suitably regularized Brown-York stress-energy tensor. It is then natural to wonder about the fundamental degrees of freedom and the precise form of the theory from which the fluid is made of.

In our context, since Einstein gravity in 3D is devoid of local propagating degrees of freedom, the identification of the fundamental degrees of freedom at the boundary can be completely performed. Indeed, the asymptotic behavior of the Einstein equations, with the boundary conditions in [6], is such that they reduce to the equations of the KdV hierarchy to all orders, i.e., without the need of performing a (hydrodynamic) derivative expansion at the boundary. Remarkably, the dynamics of the non-linear fluid, that evolves according to the KdV equations, was shown to emerge from the conservation law of left and right momentum densities, where the underlying fields are manifestly unveiled and fulfill the potential modified $\mathrm{KdV}$ equations.

As an ending remark, it is worth mentioning that different classes of boundary conditions relating pure three-dimensional gravity with integrable systems have been proposed in [33-36]. It would then be interesting to explore whether a similar construction, as the one performed here, could be carried out in those cases, as well as in the context of higher spin gravity with different set of boundary conditions as in [6, 37-39].

\section{Acknowledgments}

We thank Oscar Fuentealba, Wout Merbis, Alfredo Pérez, Miguel Riquelme and David Tempo, for useful discussions and comments. The work of H.G. is supported by the Austrian Science Fund (FWF), project P 28751-N2 and P 27182-N27. The work of J.M. was supported by the ERC Advanced Grant "High-Spin-Grav", by FNRS-Belgium (convention FRFC PDR T.1025.14 and convention IISN 4.4503.15). This research has been partially supported by FONDECYT grants $\mathrm{N}^{\circ} 1161311,1171162,1181496,1181628$, and the grant CONICYT PCI/REDES 170052. The Centro de Estudios Científicos (CECs) is funded by the Chilean Government through the Centers of Excellence Base Financing Program of Conicyt. 


\section{A Gelfand-Dikii polynomials and Hamiltonians}

The Gelfand-Dikii polynomials can be constructed from the recurrence relation (2.13). In our conventions, the first five polynomials are explicitly given by

$$
\begin{aligned}
R_{0}^{ \pm}= & 1 \\
R_{1}^{ \pm}= & \mathcal{L}^{ \pm} \\
R_{2}^{ \pm}= & \frac{3}{2} \mathcal{L}^{ \pm 2}-\frac{k}{4 \pi} \partial_{\phi}^{2} \mathcal{L}^{ \pm} \\
R_{3}^{ \pm}= & \frac{5}{2} \mathcal{L}^{ \pm 3}-\frac{5 k}{8 \pi}\left(\partial_{\phi} \mathcal{L}^{ \pm}\right)^{2}-\frac{5 k}{4 \pi} \mathcal{L}^{ \pm} \partial_{\phi}^{2} \mathcal{L}^{ \pm}+\frac{k^{2}}{16 \pi^{2}} \partial_{\phi}^{4} \mathcal{L}^{ \pm} \\
R_{4}^{ \pm}= & \frac{35}{8} \mathcal{L}^{ \pm 4}-\frac{35 k}{8 \pi} \mathcal{L}^{ \pm}\left(\partial_{\phi} \mathcal{L}^{ \pm}\right)^{2}-\frac{35 k}{8 \pi} \mathcal{L}^{ \pm} \partial_{\phi}^{2} \mathcal{L}^{ \pm}+\frac{21 k^{2}}{32 \pi^{2}}\left(\partial_{\phi}^{2} \mathcal{L}^{ \pm}\right)^{2} \\
& +\frac{7 k^{2}}{8 \pi^{2}}\left(\partial_{\phi} \mathcal{L}^{ \pm}\right)\left(\partial_{\phi}^{3} \mathcal{L}^{ \pm}\right)+\frac{7 k^{2}}{16 \pi^{2}} \mathcal{L}^{ \pm} \partial_{\phi}^{4} \mathcal{L}^{ \pm}-\frac{k^{3}}{64 \pi^{3}} \partial_{\phi}^{6} \mathcal{L}^{ \pm}
\end{aligned}
$$

Their corresponding densities (2.15) then read

$$
\begin{aligned}
\mathcal{H}_{0}^{ \pm} & =\mathcal{L}^{ \pm} \\
\mathcal{H}_{1}^{ \pm} & =\frac{1}{2} \mathcal{L}^{ \pm 2}, \\
\mathcal{H}_{2}^{ \pm} & =\frac{1}{2} \mathcal{L}^{ \pm 3}+\frac{k}{8 \pi}\left(\partial_{\phi} \mathcal{L}^{ \pm}\right)^{2}, \\
\mathcal{H}_{3}^{ \pm} & =\frac{5}{8} \mathcal{L}^{ \pm 4}+\frac{5 k}{8 \pi} \mathcal{L}^{ \pm}\left(\partial_{\phi} \mathcal{L}^{ \pm}\right)^{2}+\frac{k^{2}}{32 \pi^{2}}\left(\partial_{\phi}^{2} \mathcal{L}^{ \pm}\right)^{2}, \\
\mathcal{H}_{4}^{ \pm} & =\frac{7}{8} \mathcal{L}^{ \pm 5}+\frac{35 k}{16 \pi} \mathcal{L}^{ \pm 2}\left(\partial_{\phi} \mathcal{L}^{ \pm}\right)^{2}+\frac{7 k^{2}}{32 \pi^{2}} \mathcal{L}^{ \pm}\left(\partial_{\phi}^{2} \mathcal{L}^{ \pm}\right)^{2}+\frac{k^{3}}{128 \pi^{3}}\left(\partial_{\phi}^{3} \mathcal{L}^{ \pm}\right)^{2}
\end{aligned}
$$

Thus, according to eq. (2.7), with $\mu^{ \pm}=R_{n}^{ \pm}$, the first four equations of the KdV hierarchy are given by

$$
\begin{aligned}
\pm \ell \partial_{t} \mathcal{L}^{ \pm}= & \partial_{\phi} \mathcal{L}^{ \pm} \\
\pm \ell \partial_{t} \mathcal{L}^{ \pm}= & 3 \mathcal{L}^{ \pm} \partial_{\phi} \mathcal{L}^{ \pm}-\frac{k}{4 \pi} \partial_{\phi}^{3} \mathcal{L}^{ \pm} \\
\pm \ell \partial_{t} \mathcal{L}^{ \pm}= & \frac{15}{2} \mathcal{L}^{ \pm 2} \partial_{\phi} \mathcal{L}^{ \pm}-\frac{5 k}{2 \pi} \partial_{\phi} \mathcal{L}^{ \pm} \partial_{\phi}^{2} \mathcal{L}^{ \pm}-\frac{5 k}{4 \pi} \mathcal{L}^{ \pm} \partial_{\phi}^{3} \mathcal{L}^{ \pm}+\frac{k^{2}}{16 \pi^{2}} \partial_{\phi}^{5} \mathcal{L}^{ \pm} \\
\pm \ell \partial_{t} \mathcal{L}^{ \pm}= & \frac{35}{2} \mathcal{L}^{ \pm} \partial_{\phi} \mathcal{L}^{ \pm}-\frac{35 k}{8 \pi}\left(\partial_{\phi} \mathcal{L}^{ \pm}\right)^{3}-\frac{35 k}{2 \pi} \mathcal{L}^{ \pm} \partial_{\phi} \mathcal{L}^{ \pm} \partial_{\phi}^{2} \mathcal{L}^{ \pm}-\frac{35 k}{8 \pi} \mathcal{L}^{ \pm 2} \partial_{\phi}^{3} \mathcal{L}^{ \pm} \\
& +\frac{35 k^{2}}{16 \pi^{2}} \partial_{\phi}^{2} \mathcal{L}^{ \pm} \partial_{\phi}^{3} \mathcal{L}^{ \pm}+\frac{21 k^{2}}{16 \pi^{2}} \partial_{\phi} \mathcal{L}^{ \pm} \partial_{\phi}^{4} \mathcal{L}^{ \pm}+\frac{7 k^{2}}{16 \pi^{2}} \mathcal{L}^{ \pm} \partial_{\phi}^{5} \mathcal{L}^{ \pm}-\frac{k^{3}}{64 \pi^{3}} \partial_{\phi}^{7} \mathcal{L}^{ \pm}
\end{aligned}
$$




\section{B pmKdV equations}

The first four equations of the potential modified KdV hierarchy read

$$
\begin{aligned}
& \pm \ell \dot{\varphi}_{ \pm}=\varphi_{ \pm}^{\prime} \\
& \pm \ell \dot{\varphi}_{ \pm}=\frac{k}{4 \pi}\left(\frac{1}{2} \varphi_{ \pm}^{\prime 3}-\varphi_{ \pm}^{\prime \prime \prime}\right), \\
& \pm \ell \dot{\varphi}_{ \pm}=\left(\frac{k}{4 \pi}\right)^{2}\left(\frac{3}{8} \varphi_{ \pm}^{\prime 5}-\frac{5}{2} \varphi_{ \pm}^{\prime} \varphi_{ \pm}^{\prime \prime 2}-\frac{5}{2} \varphi_{ \pm}^{\prime 2} \varphi_{ \pm}^{\prime \prime \prime}+\varphi_{ \pm}^{(5)}\right) \text {, } \\
& \pm \ell \dot{\varphi}_{ \pm}=\left(\frac{k}{4 \pi}\right)^{3}\left(\frac{5}{16} \varphi_{ \pm}^{\prime 7}-\frac{35}{4} \varphi_{ \pm}^{\prime}{ }^{3} \varphi_{ \pm}^{\prime \prime 2}-\frac{35}{8} \varphi_{ \pm}^{\prime 4} \varphi_{ \pm}^{\prime \prime \prime}+\frac{35}{2} \varphi_{ \pm}^{\prime \prime 2} \varphi_{ \pm}^{\prime \prime \prime}+\frac{21}{2} \varphi_{ \pm}^{\prime} \varphi_{ \pm}^{\prime \prime \prime}\right. \\
& \left.+14 \varphi_{ \pm}^{\prime} \varphi_{ \pm}^{\prime \prime} \varphi_{ \pm}^{\prime \prime \prime \prime}+\frac{7}{2} \varphi_{ \pm}^{\prime 2} \varphi_{ \pm}^{(5)}-\varphi_{ \pm}^{(7)}\right) \text {. }
\end{aligned}
$$

Open Access. This article is distributed under the terms of the Creative Commons Attribution License (CC-BY 4.0), which permits any use, distribution and reproduction in any medium, provided the original author(s) and source are credited.

\section{References}

[1] J.D. Brown and M. Henneaux, Central Charges in the Canonical Realization of Asymptotic Symmetries: An Example from Three-Dimensional Gravity, Commun. Math. Phys. 104 (1986) 207 [INSPIRE].

[2] O. Coussaert, M. Henneaux and P. van Driel, The Asymptotic dynamics of three-dimensional Einstein gravity with a negative cosmological constant, Class. Quant. Grav. 12 (1995) 2961 [gr-qc/9506019] [INSPIRE].

[3] M. Henneaux, L. Maoz and A. Schwimmer, Asymptotic dynamics and asymptotic symmetries of three-dimensional extended AdS supergravity, Annals Phys. 282 (2000) 31 [hep-th/9910013] [inSPIRE].

[4] G. Barnich, H.A. González and P. Salgado-Rebolledo, Geometric actions for three-dimensional gravity, Class. Quant. Grav. 35 (2018) 014003 [arXiv:1707.08887] [INSPIRE].

[5] J. Cotler and K. Jensen, A theory of reparameterizations for $A d S_{3}$ gravity, arXiv: 1808.03263 [INSPIRE].

[6] A. Pérez, D. Tempo and R. Troncoso, Boundary conditions for General Relativity on AdS 3 and the KdV hierarchy, JHEP 06 (2016) 103 [arXiv: 1605.04490] [INSPIRE].

[7] A. Achucarro and P.K. Townsend, A Chern-Simons Action for Three-Dimensional anti-de Sitter Supergravity Theories, Phys. Lett. B 180 (1986) 89 [InSPIRE].

[8] E. Witten, (2+1)-Dimensional Gravity as an Exactly Soluble System, Nucl. Phys. B 311 (1988) 46 [INSPIRE].

[9] P. Forgacs, A. Wipf, J. Balog, L. Feher and L. O'Raifeartaigh, Liouville and Toda Theories as Conformally Reduced WZNW Theories, Phys. Lett. B 227 (1989) 214 [inSPIRE].

[10] A. Alekseev and S.L. Shatashvili, Path Integral Quantization of the Coadjoint Orbits of the Virasoro Group and 2D Gravity, Nucl. Phys. B 323 (1989) 719 [INSPIRE]. 
[11] M. Henneaux, A. Pérez, D. Tempo and R. Troncoso, Chemical potentials in three-dimensional higher spin anti-de Sitter gravity, JHEP 12 (2013) 048 [arXiv:1309.4362] [INSPIRE].

[12] C. Bunster, M. Henneaux, A. Pérez, D. Tempo and R. Troncoso, Generalized Black Holes in Three-dimensional Spacetime, JHEP 05 (2014) 031 [arXiv: 1404.3305] [INSPIRE].

[13] I.M. Gelfand and L.A. Dikii, Asymptotic behavior of the resolvent of Sturm-Liouville equations and the algebra of the Korteweg-de Vries equations, Russ. Math. Surveys 30 (1975) 77 [INSPIRE].

[14] T. Regge and C. Teitelboim, Role of Surface Integrals in the Hamiltonian Formulation of General Relativity, Annals Phys. 88 (1974) 286 [INSPIRE].

[15] M. Bañados, C. Teitelboim and J. Zanelli, The Black hole in three-dimensional space-time, Phys. Rev. Lett. 69 (1992) 1849 [hep-th/9204099] [InSPIRE].

[16] M. Bañados, M. Henneaux, C. Teitelboim and J. Zanelli, Geometry of the $(2+1)$ black hole, Phys. Rev. D 48 (1993) 1506 [Erratum ibid. D 88 (2013) 069902] [gr-qc/9302012] [INSPIRE].

[17] M. Rooman and P. Spindel, Holonomies, anomalies and the Fefferman-Graham ambiguity in $A d S_{3}$ gravity, Nucl. Phys. B 594 (2001) 329 [hep-th/0008147] [INSPIRE].

[18] G. Barnich and H.A. González, Dual dynamics of three dimensional asymptotically flat Einstein gravity at null infinity, JHEP 05 (2013) 016 [arXiv:1303.1075] [INSPIRE].

[19] G. Barnich and G. Compere, Classical central extension for asymptotic symmetries at null infinity in three spacetime dimensions, Class. Quant. Grav. 24 (2007) F15 [gr-qc/0610130] [INSPIRE].

[20] E. Witten, Quantum Field Theory and the Jones Polynomial, Commun. Math. Phys. 121 (1989) 351 [INSPIRE].

[21] S. Elitzur, G.W. Moore, A. Schwimmer and N. Seiberg, Remarks on the Canonical Quantization of the Chern-Simons-Witten Theory, Nucl. Phys. B 326 (1989) 108 [InSPIRE].

[22] P.J. Olver, Applications of Lie groups to differential equations, vol. 107, Springer Science \& Business Media (2000).

[23] J.P. Wang, A list of $1+1$ dimensional integrable equations and their properties, J. Nonlinear Math. Phys. 9 (2002) 213.

[24] R. Floreanini and R. Jackiw, Selfdual Fields as Charge Density Solitons, Phys. Rev. Lett. 59 (1987) 1873 [INSPIRE].

[25] S.A. Hartnoll, Lectures on holographic methods for condensed matter physics, Class. Quant. Grav. 26 (2009) 224002 [arXiv:0903.3246] [INSPIRE].

[26] H.A. González, D. Tempo and R. Troncoso, Field theories with anisotropic scaling in 2D, solitons and the microscopic entropy of asymptotically Lifshitz black holes, JHEP 11 (2011) 066 [arXiv: 1107.3647] [INSPIRE].

[27] M. Taylor, Lifshitz holography, Class. Quant. Grav. 33 (2016) 033001 [arXiv:1512.03554] [INSPIRE].

[28] H.P. McKean and P. van Moerbeke, The spectrum of hill's equation, Invent. Math. 30 (1975) 217.

[29] B.A. Dubrovin, V.B. Matveev and S.P. Novikov, Non-linear equations of Korteweg-De Vries type, finite-zone linear operators, and abelian varieties, Russ. Math. Surv. 31 (1976) 59. 
[30] M. Rangamani, Gravity and Hydrodynamics: Lectures on the fluid-gravity correspondence, Class. Quant. Grav. 26 (2009) 224003 [arXiv:0905.4352] [INSPIRE].

[31] V.E. Hubeny, The Fluid/Gravity Correspondence: a new perspective on the Membrane Paradigm, Class. Quant. Grav. 28 (2011) 114007 [arXiv:1011.4948] [INSPIRE].

[32] I. Bredberg, C. Keeler, V. Lysov and A. Strominger, From Navier-Stokes To Einstein, JHEP 07 (2012) 146 [arXiv: 1101.2451] [INSPIRE].

[33] H. Afshar et al., Soft Heisenberg hair on black holes in three dimensions, Phys. Rev. D 93 (2016) 101503 [arXiv: 1603.04824] [INSPIRE].

[34] H. Afshar, D. Grumiller, W. Merbis, A. Pérez, D. Tempo and R. Troncoso, Soft hairy horizons in three spacetime dimensions, Phys. Rev. D 95 (2017) 106005 [arXiv:1611.09783] [INSPIRE].

[35] O. Fuentealba et al., Integrable systems with $B M S_{3}$ Poisson structure and the dynamics of locally flat spacetimes, JHEP 01 (2018) 148 [arXiv:1711.02646] [INSPIRE].

[36] D. Melnikov, F. Novaes, A. Pérez and R. Troncoso, Lifshitz Scaling, Microstate Counting from Number Theory and Black Hole Entropy, arXiv:1808.04034 [INSPIRE].

[37] G. Compère and W. Song, $\mathcal{W}$ symmetry and integrability of higher spin black holes, JHEP 09 (2013) 144 [arXiv: 1306.0014] [INSPIRE].

[38] M. Gutperle and Y. Li, Higher Spin Lifshitz Theory and Integrable Systems, Phys. Rev. D 91 (2015) 046012 [arXiv: 1412.7085] [INSPIRE].

[39] M. Beccaria, M. Gutperle, Y. Li and G. Macorini, Higher spin Lifshitz theories and the Korteweg-de Vries hierarchy, Phys. Rev. D 92 (2015) 085005 [arXiv:1504.06555] [InSPIRE]. 